\title{
Microgel Swelling Studied by Cryo-SEM
}

\author{
Jing Liang ${ }^{1}$, Feiyue Teng ${ }^{1}$, Tseng-Ming $\mathrm{Chou}^{2}$ and Matthew Libera ${ }^{1}$ \\ 1. Dept. of Chemical Engr. \& Materials Science, Stevens Institute of Technology, Hoboken, NJ USA \\ 2. Laboratory for Multiscale Imaging, Stevens Institute of Technology, Hoboken, NJ USA
}

The swell ratio is an essential feature of hydrogels. It reflects intrinsic network structure, which controls hydrogel transport and mechanical properties [1-2]. Microgels are micro-sized hydrogels that possess unique properties associated with surface modification and with their use as delivery vehicles. However, their small size hinders characterization of microgel swell ratio by the traditional weighing methods used for macroscopic gels. This study takes advantage of cryo-SEM to characterize the microgel swell ratio and reveals microgel morphology in the hydrated state.

Here, poly(ethylene glycol)-co-(acrylic acid) microgels were suspended in $0.01 \mathrm{M}$ phosphate buffer at pH 3 and $\mathrm{pH} 9$ and were then frozen by high-pressure freezing (Leica HPM-100 HPF). The frozen samples were fractured and imaged using a Zeiss Auriga Cross-Beam FIB-SEM equipped with a Leica VCT-100 cryo-transfer system. Samples were warmed to $-95^{\circ} \mathrm{C}$ for sublimation and were cooled to $135^{\circ} \mathrm{C}$ for SEM imaging. Different degrees of ice removal were obtained by varying the sublimation time: no sublimation; light sublimation (10 min); strong sublimation (40 min); full sublimation (12 h).

Figure 1 shows SEM images of microgels suspended in $\mathrm{pH} 3$ phosphate buffer that underwent different degrees of sublimation. Fig. 1A is an image of microgels with no sublimation and shows only the crosssection of fractured microgels and the surrounding ice. Subsequent sublimation reveals the spherical structure of microgels (Fig. 1B-D). A depth of approximately $1 \mu \mathrm{m}$ of surrounding ice was removed after 10 min of sublimation (Fig. 1B). When sublimed for more than 40 min (Fig. 1C-D), the overall spherical morphology of microgels was imaged. Under these sublimation conditions, full microgel diameters could be directly measured when more than half of the microgel hemisphere was shown. The water content within the microgels was monitored by the $\mathrm{C} / \mathrm{O}$ X-ray intensity ratio measured at different stages of sublimation by EDS. These measurements show that microgels remained hydrated even after strong sublimation but are fully dried after full sublimation. The hydrated and dried microgel diameters were obtained under the strong and full sublimation conditions, respectively. Swell-ratio results are summarized in Table 1. The dried microgel diameters of $\mathrm{pH} 3$ and $\mathrm{pH} 9$ agree well. This result substantiates the accuracy of measuring microgel sizes through this method. The hydrated diameters of microgels at $\mathrm{pH} 9$ are larger than those at $\mathrm{pH} 3$, which is consistent with the deprotonation of acrylic acid at a $\mathrm{pH}$ above its $\mathrm{pKa}$ (4.5). The volume swell ratios were calculated based on the dried and hydrated diameters. Importantly, the microgel swell ratios measured by cryo-SEM are almost the same as those of identical macroscopic gels using weight measurements [3].

\section{References:}

[1] Paul J. Flory and John Rehner Jr, The Journal of Chemical Physics 11 (1943), p. 512.

[2] Paolo Colombo, Advanced Drug Delivery Reviews 11 (1993), p. 37.

[3] This work has been supported by the United States National Science Foundation (grant \#DMR1608406) and used microscopy resources supported by the National Science Foundation (grants \#DMR1428296 and DMR-0922522) and the Stevens Institute of Technology. 


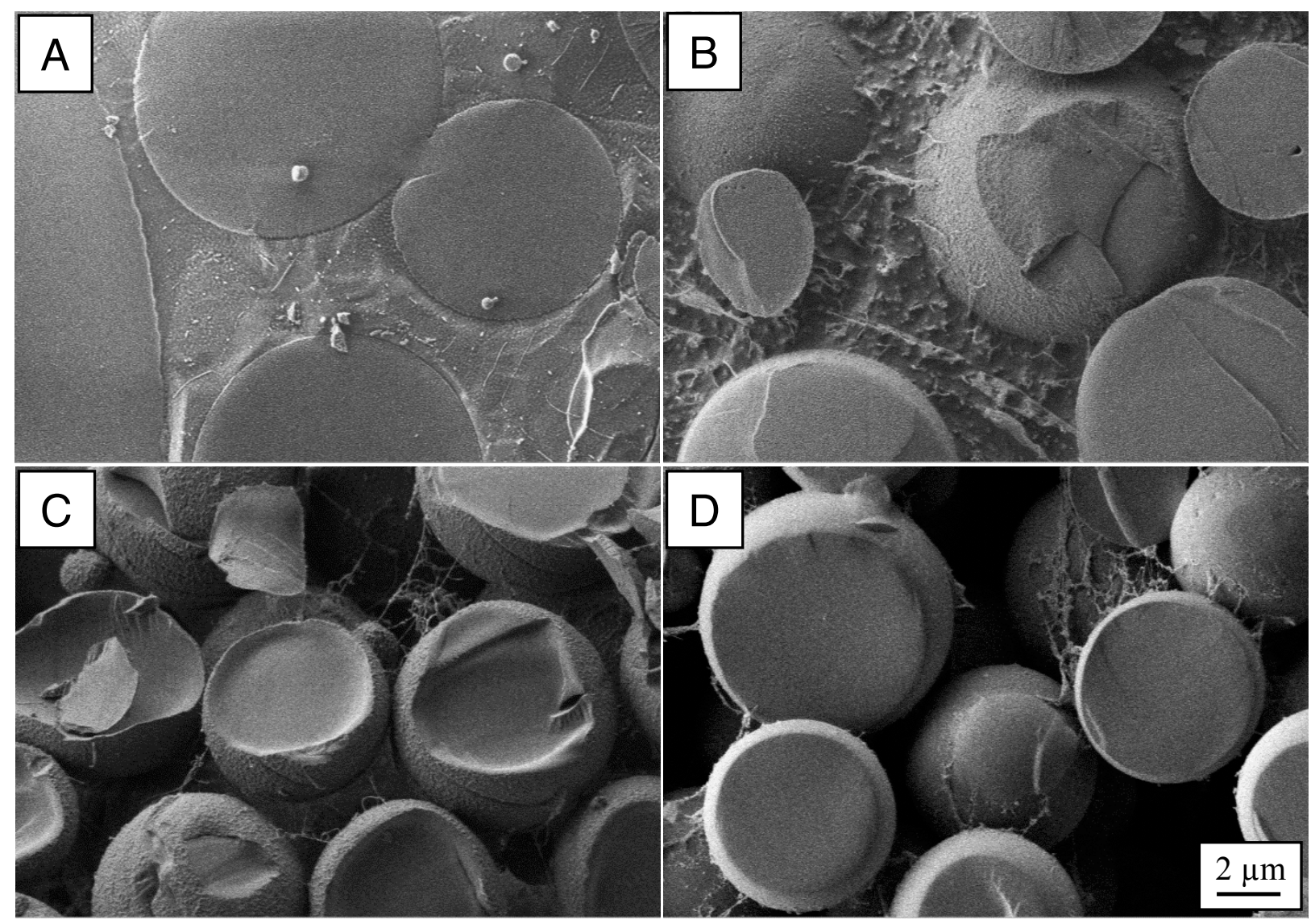

Figure 1. SEM images of microgels after different sublimation times: (A) no sublimation; (B) light (10 min) sublimation; (C) strong (30 min) sublimation; and (D) full sublimation. A, $\mathrm{B}$, and $\mathrm{C}$ are SEM images taken at $-135^{\circ} \mathrm{C}$ while $\mathrm{D}$ is an image taken at room temperature.

Table 1. Diameters and swell ratios of microgels at $\mathrm{pH} 3$ and $\mathrm{pH} 9$

\begin{tabular}{lll}
\hline & pH 3 & pH 9 \\
\hline Dried $/ \mu \mathrm{m}$ & $6.2 \pm 0.6$ & $6.5 \pm 0.8$ \\
Hydrated $/ \mu \mathrm{m}$ & $9.2 \pm 0.8$ & $10.3 \pm 1.3$ \\
Swell ratio & 3.3 & 4.0 \\
\hline
\end{tabular}

